\title{
An Endoreversible Thermodynamic Model Applied to the Convective Zone of the Sun
}

\author{
J. Ortuño-Araujo, ${ }^{1}$ M. A. Barranco-Jiménez, ${ }^{1}$ Janos Zsargo, $^{2}$ and F. Angulo-Brown ${ }^{2}$ \\ ${ }^{1}$ Departamento de Formación Básica, Escuela Superior de Cómputo del IPN, Avenue Miguel Bernard s/n., Esquire Juan de Dios Bátiz, \\ U.P. Zacatenco, 07738 México, D F, Mexico \\ ${ }^{2}$ Departamento de Física, Escuela Superior de Física y Matemáticas del IPN, Edif. 9, U.P. Zacatenco, 07738 México, D F, Mexico
}

Correspondence should be addressed to F. Angulo-Brown, angulo@esfm.ipn.mx

Received 22 December 2011; Accepted 22 January 2012

Academic Editors: M. Ding and A. Pamyatnykh

Copyright (๑) 2012 J. Ortuño-Araujo et al. This is an open access article distributed under the Creative Commons Attribution License, which permits unrestricted use, distribution, and reproduction in any medium, provided the original work is properly cited.

\begin{abstract}
Within the context of finite-time thermodynamics (FTTs) some models of convective atmospheric cells have been proposed to calculate the efficiency of the conversion of solar energy into wind energy and also for calculating the surface temperature of the planets of the solar system. One of these models is the Gordon and Zarmi (GZ) model, which consists in taking the sun-earthwind system as a FTT-cyclic heat engine where the heat input is solar radiation, the working fluid is the earth's atmosphere and the energy in the winds is the work produced. The cold reservoir to which the engine rejects heat is the $3 \mathrm{~K}$ surrounding universe. In the present work we apply the GZ-model to investigate some features of the convective zone of the sun by means of a possible structure of successive convective cells along the well-established convective region of the sun. That is, from $0.714 R_{S}$ up to $R_{S}$ being $R_{S}$ the radius of the sun. Besides, we estimate the number of cells of the model, the possible size of the cells, their thermal efficiency, and also their average power output. Our calculations were made by means of two FTT regimes of performance: the maximum power regime and the maximum ecological function regime. Our results are in reasonable agreement with others reported in the literature.
\end{abstract}

\section{Introduction}

The problem of thermal balance between the planets of the solar system and the sun under a finite-time thermodynamics approach has been treated by several authors [1-8]. In some of these articles the question of the conversion of solar energy into wind energy is also treated. In particular, De Vos [3] demonstrated that cosmic radiation, starlight, and moonlight can be neglected for the thermal balance of any of the planets of the solar system and only the following quantities have an influence: the incident solar influx or solar constant $I_{\mathrm{sc}}$, the planet's albedo $\rho$, and the greenhouse effect of the planet's atmosphere crudely evaluated by means of a coefficient $\gamma$. This coefficient can be taken as the normalized greenhouse effect introduced by Raval and Ramanathan in [9]. When only the global thermal balance between the sun and a planet is considered, one can roughly obtain the planet's surface temperature assumed as a uniform temperature $T_{p}$. If the conversion of solar energy into wind energy is to be modeled, it is necessary to involve at least two representative atmospheric temperatures for making the creation of work possible; that is, to take the planet's atmosphere as a working fluid that converts heat into mechanical work. In 1989, Gordon and Zarmi [1] introduced a FTT-model taking the sun-earth-wind system as a FTT-cyclic heat engine where the heat input is solar radiation, the working fluid is the earth's atmosphere, and the energy in the winds is the work produced; the cold reservoir to which the engine rejects heat is the $3 \mathrm{~K}$ surrounding universe. By means of this simplified model, Gordon and Zarmi were able to obtain reasonable values for the annual average power in the earth's winds and for the average maximum and minimum temperatures of the atmosphere, without resorting to detailed dynamic models of the earth's atmosphere, and without considering any other effect (such as earth's rotation, earth's orbital motion around 
the Sun, and ocean currents). Later, De Vos and Flater [2] extended the GZ model to take into account the wind energy dissipation by means of a maximum power criterion. This model was extended by De Vos and van der Wel $[4,5]$ by constructing a model based in convective Hadley cells. All the models used in [1-5] are endoreversible ones in the sense of FTT [10], that is, all irreversibilities are located in the exchanges between the engine and the external world. The GZ model was later studied under a nonendoreversible approach and by using the so-called ecological optimization criterion $[6,7]$. This approach [11] consists of maximizing a function $E$ that represents a good compromise between highpower output and low-entropy production. The function $E$ is given by

$$
E=P-T_{\text {ext }} \Delta S_{u}
$$

where $P$ is the power output of the cycle, $\Delta S_{u}$ the total entropy production (system plus surroundings) per cycle, and $T_{\text {ext }}$ is the temperature of the cold reservoir. This optimization criterion for the case of the so-called CurzonAhlborn cycle [12], for instance, leads to a cycle configuration such that for maximum $E$ it produces around $75 \%$ of the maximum power and only about $25 \%$ of the entropy produced in the maximum power regime [13]. By means of employing this criterion in a nonendoreversible GZ model, the authors of [6] also found reasonable values for the annual average power of the winds and for the extreme temperatures of the earth's troposphere. Later, the nonendoreversible GZ model was applied to calculate the surface temperature of planets of the solar system [8], considering two regimes of performance: maximum power regime and maximum ecological function regime. In this work, we apply the GZ model to the convective zone of the sun which is located between $0.714 R_{S}$ and $R_{S}$ [14]. Our FTT approach leads to a possible structure of the convective region of the sun consisting in approximately sixteen coupled cells. It is important to remark that these sixteen convective Carnotian cells are only a kind of idealized cells, thermodynamically equivalent to the complex structure of the actual convective zone of the sun. The paper is organized as follows: in Section 2, we present a brief review of the GZ model for the convective cells under both the maximum power and the ecological function regimes. In Section 3, we applied the GZ model to the convective zone of the sun and finally in Section 4 we present some concluding remarks.

\section{Endoreversible GZ Model for Atmospheric Convection}

The endoreversible GZ model is based on annual average quantities and thus it does not represent actual convective cells but a kind of annual virtual cell that takes into account the global thermodynamic restrictions over the convection as a dominant energy transfer mechanism in the air (which has a large Rayleigh number). Besides, this kind of model must only be taken as one that producing better upper bounds than those calculated by means of classical equilibrium thermodynamics, which is one of the main purposes of FTT.

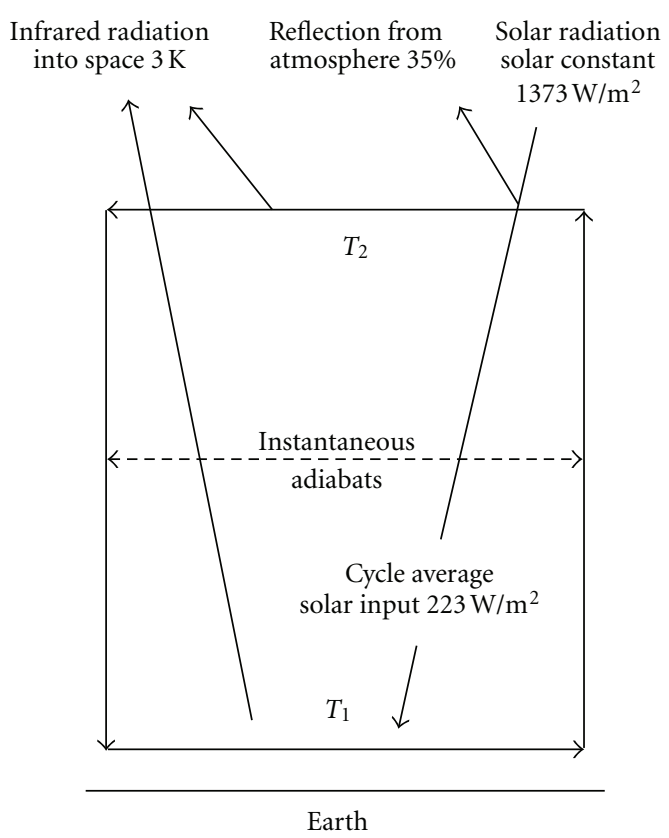

FIGURE 1: Scheme of a simplified solar-driven heat engine (taken from [1]).

2.1. Maximum Power Regime. In Figure 1, a schematic view of a simplified sun-earth-winds system as a heat engine cycle is depicted. This cycle consists of four branches: (1) two isothermal branches, one in which the atmosphere absorbs solar radiation at low altitudes and one in which the atmosphere rejects heat at high altitudes to the universe and (2) two intermediate instantaneous adiabats [10] with rising and falling currents. In [15], it was shown that a Curzon-Ahlborn FTT cycle in the endoreversible limit with instantaneous adiabats is reached for large compression ratios. In the GZ virtual cells, it is feasible to consider that this condition is fulfilled. According to GZ, this oversimplified Carnot-like engine corresponds very approximately to the global scale motion of wind in convective cells. Below, we use all of GZ model's assumptions.

For example, the work performed by the working fluid in one cycle $W$, the internal energy of the working fluid $U$, and the yearly average solar radiation flux $q_{s}$ are expressed per unit area of the earth's surface. The temperatures of the four-branch cycle are taken as follows: $T_{1}$ is the working fluid temperature in the isothermal branch at the lowest altitude, where the working fluid absorbs solar radiation for half of the cycle. During the second half of the cycle, heat is rejected via black-body radiation from the working fluid at temperature $T_{2}$ (highest altitude of the cell) to the cold reservoir at temperature $T_{\text {ext }}$ (the surrounding $3 \mathrm{~K}$ universe). In the GZ model, the objective is to maximize the work per cycle (average power) subjected to the endoreversibility constraint [10], that is,

$$
\Delta S_{\text {int }}=\int_{0}^{t_{o}}\left\{\frac{q_{s}(t)-\sigma\left[T^{4}(t)-T_{\mathrm{ext}}^{4}(t)\right]}{T(t)}\right\} d t=0,
$$


where $\Delta S_{\text {int }}$ is the change of entropy per unit area, $t_{0}$ is the time of one cycle, $\sigma$ is the Stefan-Boltzmann constant (5.67 $\times$ $10^{-8} \mathrm{~W} / \mathrm{m}^{2} \mathrm{~K}^{4}$ ), and $q_{s}$, and $T$ are functions of time $t$, taken as [1]

$$
\begin{gathered}
T(t)= \begin{cases}T_{1} ; & \text { if } 0 \leq t \leq \frac{t_{0}}{2}, \\
T_{2} ; & \text { if } \frac{t_{0}}{2} \leq t \leq t_{0},\end{cases} \\
q_{s}(t)= \begin{cases}0 ; & \text { if } \frac{t_{0}}{2} \leq t \leq t_{0}, \\
\frac{I_{\mathrm{sc}}(1-\rho)}{2} ; & \text { if } 0 \leq t \leq \frac{t_{0}}{2},\end{cases}
\end{gathered}
$$

in the same way, $T_{\text {ext }}=3 \mathrm{~K}$ for $0 \leq t \leq t_{0}$, with $I_{\text {sc }}$ the yearly average solar constant $\left(1373 \mathrm{~W} / \mathrm{m}^{2}\right)$ and $\rho=0.35$ [2], the effective average albedo of the earth's atmosphere. The GZ model maximizes the work per cycle $W$, taken from the first law of thermodynamics:

$$
\Delta U=-W+\int_{0}^{t_{0}}\left\{q_{s}(t)-\sigma\left[T^{4}(t)-T_{\mathrm{ext}}^{4}(t)\right]\right\} d t=0,
$$

by denoting average values as,

$$
\bar{T}=\frac{T_{1}+T_{2}}{2}, \quad \overline{T^{n}}=\frac{T_{1}^{n}+T_{2}^{n}}{2}, \quad \overline{q_{s}}=I_{s c} \frac{(1-\rho)}{4},
$$

where $n$ is an integer with values $n=3$ or 4 . The factor of $1 / 4$ arises from a factor of $1 / 2$ to account for the day/night difference and a geometric factor of $1 / 2$ to account for the earth's cross section, which is intercepted by solar radiation, as opposed to the corresponding hemispherical surface area of the earth. From (4) and (5) and taking into account the constraint given by (2), GZ construct the following Lagrangian $L$;

$$
L=T^{4}(t)+\lambda\left[\frac{q_{s}(t)}{T(t)}-\sigma T^{3}(t)\right],
$$

where $\lambda$ is a Lagrange multiplier. The Euler-Lagrange formalism will be used, by using $\partial L(t) / \partial T(t)=0$, GZ found the following values for the earth's atmosphere: $T_{1}=277 \mathrm{~K}$, $T_{2}=192 \mathrm{~K}$, and $P_{\max }=W_{\max } / t_{0}=17.1 \mathrm{~W} / \mathrm{m}^{2}$. These numerical values are not so far from "actual" values, which are $P \approx 7 \mathrm{~W} / \mathrm{m}^{2}$ [16], $T_{1}=290 \mathrm{~K}$ (at ground level), and $T_{2} \approx 195 \mathrm{~K}$ (at an altitude of around $75-90 \mathrm{Km}$ ). However, as GZ assert, their power calculation must be taken as an upper bound due to several idealizations in their model. In [6], another endoreversible case was analyzed in which the tropopause layer with $T_{\text {ext }}=200 \mathrm{~K}$ was used as cold reservoir. In this case, the following Lagrangian was used:

$$
\begin{aligned}
L(t)= & \overline{q_{s}+\sigma T_{\mathrm{ext}}^{4}}-\sigma \overline{T^{4}} \\
& -\alpha\left[\frac{\overline{q_{s}}}{T_{1}}-\frac{\sigma\left(T_{1}^{3}+T_{2}^{3}\right)}{2}-\sigma T_{\mathrm{ext}}^{4}\left(\frac{1}{T_{1}}+\frac{1}{T_{2}}\right)\right],
\end{aligned}
$$

with $\alpha$ a Lagrange multiplier. By numerically solving $\partial L(t) / \partial T(t)=0$, they obtained $T_{1}=293.387 \mathrm{~K}$ and $T_{2}=$ 239.267 K, which are excellent values for convective cells restricted to the troposphere. If these temperature values are substituted in the expression for the average power (see [6])

$$
P=\overline{q_{s}+\sigma T_{\mathrm{ext}}^{4}}-\sigma \overline{T^{4}},
$$

a value of $P=10.758 \mathrm{~W} / \mathrm{m}^{2}$ is obtained, which is a good value for the wind power [16].

2.2. Ecological Function Regime. As De Vos and Flater [2] state, no mechanism guarantees that the atmosphere maximizes the wind power. In fact, some authors [17-19] have recognized that the earth's atmosphere operates at nearly its maximum efficiency; thus, from an FTT point of view, an ecological-type criterion seems feasible. This is due to the properties of the $E$ function, which at its maximum value represents an austere compromise between power and entropy production, additionally leading to a high efficiency $[11,13]$. This ecological criterion, as previously occurred with the concepts of power output and efficiency [20], has also been used in the context of irreversible thermodynamics [21-23]. In particular, in [7] the so-called ecological criterion was applied to the GZ model. This criterion consists in maximizing equation (1). By means of the second law of thermodynamics, first, we calculate $\Delta S_{u}$, the total entropy change per cycle (system plus surroundings),

$$
\Delta S_{u}=\int_{0}^{t_{0}}\left\{\frac{-q_{s}(t)+\sigma\left[T^{4}(t)-T_{\mathrm{ext}}^{4}(t)\right]}{T(t)}\right\} d t .
$$

From (3), we obtain

$$
\begin{gathered}
\Delta S_{u}=\int_{0}^{t_{0} / 2}\left\{-\frac{q_{s}(t)}{T_{1}}+\sigma\left(T_{1}^{3}-\frac{T_{\mathrm{ext}}^{4}}{T_{1}}\right)\right\} d t \\
-\int_{t_{0} / 2}^{t_{0}}\left\{\sigma\left(\frac{T_{2}^{4}-T_{\mathrm{ext}}^{4}}{T_{\mathrm{ext}}}\right)\right\} d t .
\end{gathered}
$$

Thus, the total entropy production is given by $[7,8]$,

$$
\Sigma=\frac{\Delta S_{u}}{t_{0}} \approx \frac{\overline{q_{s}}}{T_{1}}+\frac{\sigma}{2}\left(T_{1}^{3}+\frac{T_{2}^{4}}{T_{\mathrm{ext}}}\right),
$$

here, we have used the approximation $\overline{q_{s}} \gg$ $\sigma T_{\text {ext }}^{4}\left(223 \mathrm{~W} / \mathrm{m}^{2} \gg 4.59 \times 10^{-6} \mathrm{~W} / \mathrm{m}^{2}\right)$ with $T_{\text {ext }}=3 \mathrm{~K}$. So, the ecological function $E$ for this case is

$$
E=\overline{q_{s}}-\sigma \overline{T^{4}}+\frac{T_{\mathrm{ext}} \overline{q_{s}}}{T_{1}}-\frac{\sigma T_{\mathrm{ext}}}{2}\left(T_{1}^{3}+\frac{T_{2}^{4}}{T_{\mathrm{ext}}}\right) .
$$

By using (12) and the constraint given by (2), we proposed the following Lagrangian function $L_{E}$ :

$$
\begin{aligned}
L_{E}= & \overline{q_{s}}-\sigma \overline{T^{4}}+\frac{T_{\mathrm{ext}} \overline{q_{s}}}{T_{1}}-\frac{\sigma T_{\mathrm{ext}}}{2} \\
& \times\left(T_{1}^{3}+\frac{T_{2}^{4}}{T_{\mathrm{ext}}}\right)-\alpha\left[\frac{\overline{q_{s}}}{T_{1}}-\sigma \overline{T^{3}}\right],
\end{aligned}
$$

with $\alpha$ being the Lagrange multiplier. By substituting the values of $\overline{q_{s}}, \sigma$, and $T_{\text {ext }}$ and numerically solving $\partial L(t) / \partial T(t)=$ 0 , we find $T_{1}=294.08 \mathrm{~K}, T_{2}=109.54 \mathrm{~K}$ and $P=6.89 \mathrm{~W} / \mathrm{m}^{2}$, 
which are reasonable values for $T_{1}$ and $P$, but not for $T_{2}$. However, if we use as a cold reservoir, the tropopause layer with $T_{\text {ext }}=200 \mathrm{~K}$, we can now use the Lagrangian function: [24],

$$
\begin{aligned}
L_{E}= & \overline{q_{s}+\sigma T_{\mathrm{ext}}^{4}}-\sigma \overline{T^{4}}+\left(\overline{q_{s}}+\frac{\sigma T_{\mathrm{ext}}^{4}}{2}\right) \frac{T_{\mathrm{ext}}}{T_{1}} \\
& -\frac{\sigma T_{\mathrm{ext}}}{2}\left(T_{1}^{3}+\frac{T_{2}^{4}}{T_{\mathrm{ext}}}\right)-\frac{\sigma T_{\mathrm{ext}}^{4}}{2} \\
& -\beta\left[\frac{\overline{q_{s}}}{T_{1}}-\frac{\sigma\left(T_{1}^{3}+T_{2}^{3}\right)}{2}+\sigma T_{\mathrm{ext}}^{4}\left(\frac{1}{T_{1}}+\frac{1}{T_{2}}\right)\right],
\end{aligned}
$$

with $\beta$ a Lagrange multiplier. By using again the EulerLagrange formalism, we numerically obtain $T_{1}=303 \mathrm{~K}$, $T_{2}=219 \mathrm{~K}$, and $P=7 \mathrm{~W} / \mathrm{m}^{2}$ which are very good values, for $T_{1}, T_{2}$, and $P$. Besides, these values are restricted to typical values in the troposphere, where the climatic phenomena occurs. It is important to note that the power values $\left(6.89 \mathrm{~W} / \mathrm{m}^{2}\right.$ and $\left.7 \mathrm{~W} / \mathrm{m}^{2}\right)$, which were calculated by the means of the ecological function, were deduced without considering the greenhouse effect ( $\gamma$ coefficient). When the later is taken into account, the values of $P$ are bigger than $7 \mathrm{~W} / \mathrm{m}^{2}[7,8]$. These scenarios lead to larger upper bounds for the wind's power permitting an energy excess for other relevant dissipative processes such as ocean currents and biological structuring.

\section{The GZ Model Applied to the Convective Zone of the Sun}

The core of the sun goes from 0 to $0.2 R_{S}$, where $R_{S}(6.96 \times$ $10^{8} \mathrm{~m} \mathrm{[14])}$ is the radius of the sun. The radiative zone embraces the region between $0.2 R_{S}$ and $0.714 R_{S}$ and beyond that lies the convective zone. The later is estimated to have a width of approximately $0.286 R_{S}$ [14]. In (8) and (11) the input data were $q_{s}$ and $T_{\text {ext }}$, the thermal energy and the temperature of the surrounding cold thermal bath for the earth's atmospheric cells, respectively. In the case of the convective zone of the sun, first we will use the maximum power criterion. In Figure 2, we show the heat fluxes balance for the convective zone of the sun. Then, by using (2), (3), (4), and (5) we obtain the following Lagrangian functional:

$$
\begin{aligned}
L\left(T_{1}, T_{2}, \lambda\right)= & \frac{q_{s}}{2}+\frac{\sigma T_{\mathrm{ext}}^{4}}{2}-\frac{\sigma}{2}\left(T_{1}^{4}+T_{2}^{4}\right) \\
& -\lambda\left[\frac{q_{s}}{2 T_{1}}-\frac{\sigma}{2}\left(T_{1}^{3}+T_{2}^{3}\right)+\frac{\sigma T_{\mathrm{ext}}^{4}}{2}\left(\frac{1}{T_{1}}+\frac{1}{T_{2}}\right)\right],
\end{aligned}
$$

where $\lambda$ is a Lagrange multiplier, $T_{\text {ext }}=3 \mathrm{~K}, T_{1}=2.18 \times 10^{6} \mathrm{~K}$ [14] is the temperature of the spherical layer at $0.714 R_{s}$ and $q_{s}=\sigma T_{1}^{4}$ the input thermal energy at the lower layer of the convective zone. The energy transport through the sun can be considered as a "sandwich", that is, there are two regions in which radiation transports the energy separated by a region where convection transports it [25]. Strictly speaking, $q_{s}$

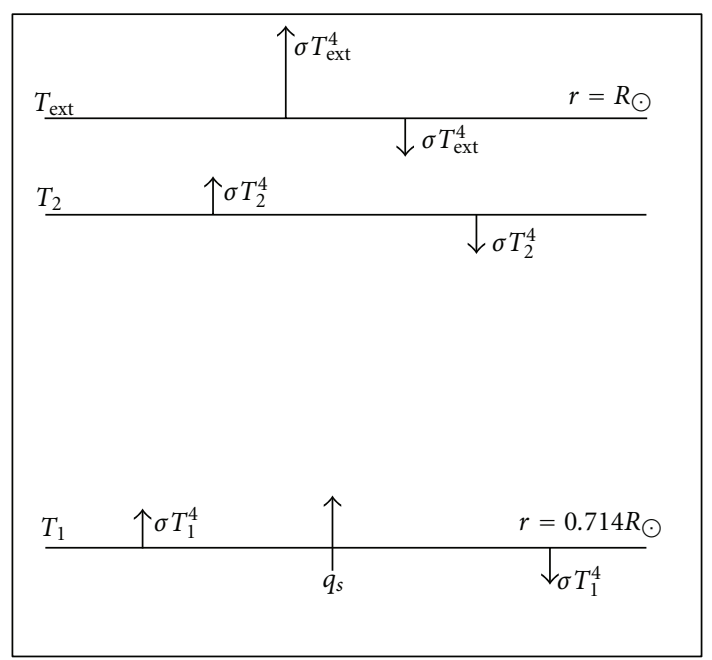

FIGURE 2: Schematic diagram of the energy fluxes present in the first internal convective cell. $T_{1}$ at $0.714 R_{s}$ is taken as the temperature of the first isothermal layer, $T_{\mathrm{ext}}=3 \mathrm{~K}$ is taken as the cold reservoir temperature, and $T_{2}$ is taken as the upper shell temperature of the first convective cell. Short arrows indicate that emitted radiation is rapidly absorbed by opaque gases.

should be calculated by means of a diffusive model based on kinetic theory of gases [25]. However, for simplicity, in our thermodynamic model we take the $0.714 R_{s}$ layer at $T_{1} \approx 2 \times 10^{6} \mathrm{~K}$ as a blackbody radiant system $\left(q_{s}=\sigma T_{1}^{4}\right.$, see Figure 2 ). The radiation emitted by this layer is rapidly absorbed by the gases at the bottom of the convective zone. For the definition of $T_{2}$, see Figure 2. By using the EulerLagrange formalism over the Lagrangian of (15), that is, $\partial L(t) / \partial T(t)=0$, we obtain the following equations:

$$
\begin{gathered}
T_{1}^{5}-\frac{\lambda}{\sigma}\left(\frac{q_{s}}{4}+\frac{3 \sigma}{4} T_{1}^{4}+\frac{\sigma T_{\mathrm{ext}}^{4}}{4}\right)=0 \\
T_{2}^{5}-\lambda\left(\frac{3}{4} T_{2}^{4}+\frac{T_{\mathrm{ext}}^{4}}{4}\right)=0 .
\end{gathered}
$$

By eliminating $\lambda$ from these equations and by using the restriction given by (2), we obtain

$$
T_{2}^{4}\left(4 T_{1}^{3} T_{2}+T_{2}^{4}-3 T_{1}^{4}\right)-T_{\mathrm{ext}}^{4}\left(T_{1}^{4}+T_{2}^{4}\right)=0 .
$$

In this equation, the only unknown variable is $T_{2}$. Then, we numerically solve (17) to obtain $T_{2}$, the temperature of the upper bound for the first convective cell starting from $T_{1}=$ $2.187761 \times 10^{6} \mathrm{~K}[14]$. Our next step is to take the obtained $T_{2}$ value of the first cell as the temperature of the lower layer of the following successive cell. This new $T_{2}$ value is taken as $T_{1}$ in (17) and then we calculate a new $T_{2}$ for the second cell. For the following successive cells we use the same recursive procedure until to reach a final $T_{2}$ coinciding approximately with the well-known value of the average surface temperature of the sun, which is $T_{S} \approx 5780 \mathrm{~K}$ [14].

In Table 1 we show that after 16 successive Carnotian convective cells we reach a final $T_{2} \approx 6000 \mathrm{~K}$. Table 1 shows 
TABle 1: Maximum power regime case: First column shows the normalized radial position of the hot layers corresponding to the sixteen virtual convective cells. The following columns give, respectively, second, the cell's widths; third, the hot isotherms; fourth, the cold isotherms; fifth, the average power output; sixth, thermal efficiency.

\begin{tabular}{lcccccc}
\hline No & $r\left(T_{1}\right) / R_{s}$ & $\Delta r=r\left(T_{2}\right)-r\left(T_{1}\right)(\mathrm{Km})$ & $T_{1} \times 10^{6}(\mathrm{~K})$ & $T_{2} \times 10^{6}(\mathrm{~K})$ & $\bar{W}\left(\mathrm{erg} / \mathrm{cm}^{2} \cdot \mathrm{s}\right)$ & $\eta=\eta\left(T_{1}, T_{2}\right)$ \\
\hline 1 & 0.714 & 61370.9 & 2.187761 & 1.51504 & $6.49519 \times 10^{17}$ & 0.307495 \\
2 & 0.802177 & 42499.6 & 1.51504 & 1.04917 & $4.65789 \times 10^{17}$ & 0.307495 \\
3 & 0.863239 & 29431.2 & 1.04917 & 0.726555 & $1.07122 \times 10^{17}$ & 0.307495 \\
4 & 0.905525 & 20381.3 & 0.726555 & 0.503143 & $2.46361 \times 10^{16}$ & 0.307495 \\
5 & 0.934809 & 14114.1 & 0.503143 & 0.348429 & $5.66582 \times 10^{15}$ & 0.307495 \\
6 & 0.955088 & 9774.09 & 0.348429 & 0.241289 & $1.30303 \times 10^{15}$ & 0.307495 \\
7 & 0.969131 & 6768.61 & 0.241289 & 0.167094 & $2.99671 \times 10^{14}$ & 0.307495 \\
8 & 0.978856 & 4687.29 & 0.167094 & 0.115713 & $6.89186 \times 10^{13}$ \\
9 & 0.985591 & 3245.97 & 0.115713 & 0.0801319 & $1.585 \times 10^{13}$ \\
10 & 0.990254 & 2247.85 & 0.0801319 & 0.0554917 & $3.64521 \times 10^{12}$ & 0.307495 \\
11 & 0.993484 & 1556.65 & 0.0554917 & 0.0384283 & $8.38353 \times 10^{11}$ & 0.307495 \\
12 & 0.995721 & 1077.99 & 0.0384283 & 0.0266118 & $1.92829 \times 10^{11}$ \\
13 & 0.99727 & 746.511 & 0.0266118 & 0.0184288 & $4.43714 \times 10^{10}$ & 0.307495 \\
14 & 0.998342 & 516.963 & 0.0184288 & 0.012762 & $1.02289 \times 10^{10}$ & 0.307495 \\
15 & 0.999085 & 357.999 & 0.012762 & 0.00883776 & $2.37682 \times 10^{9}$ & 0.307495 \\
16 & 0.999599 & 247.916 & 0.00883776 & 0.00612019 & $5.7099 \times 10^{8}$ & 0.307495 \\
\hline
\end{tabular}

our results for Carnotian cells performing in the maximum power regime. As one can see in this Table (third column), the widths of the cells are decreasing toward the outer regions. The total width is around $0.280 R_{s}$ which is not so far of the value $0.286 R_{s}$ given by other sun models [26]. If we take as the mode of thermodynamic performance of the sun's convective cells the so-called maximum ecological regime [11], in a similar way as (15), then we obtain the following Lagrangian functional:

$$
\begin{aligned}
L_{E}\left(T_{1}, T_{2}, \lambda\right)= & \frac{q_{s}}{2}\left(1-\frac{T_{\text {ext }}}{T_{1}}\right)+\sigma T_{\text {ext }}^{4} \\
& -\sigma\left(T_{1}^{4}+T_{2}^{4}\right)+\frac{\sigma T_{\text {ext }}}{2}\left(T_{1}^{3}+T_{2}^{3}\right) \\
& -\frac{\sigma T_{\text {ext }}}{2}\left(\frac{T_{1}^{4}}{T_{2}}+\frac{T_{2}^{4}}{T_{1}}\right)-\frac{\sigma T_{\text {ext }}^{5}}{2}\left(\frac{1}{T_{1}}+\frac{1}{T_{2}}\right) \\
& -\lambda\left[\frac{q_{s}}{2 T_{1}}-\frac{\sigma}{2}\left(T_{1}^{3}+T_{2}^{3}\right)-\frac{\sigma T_{\text {ext }}^{4}}{2}\left(\frac{1}{T_{1}}+\frac{1}{T_{2}}\right)\right] .
\end{aligned}
$$

By using the Euler-Lagrange formalism over the Lagrangian of (18), that is, $\partial L_{E}(t) / \partial T(t)=0$ and following a similar procedure as in the case of (17), we obtain

$$
\begin{aligned}
8 T_{\text {ext }}^{4} & T_{1} T_{2}\left(T_{1}^{4}+T_{2}^{4}\right)+3 T_{\text {ext }}^{5}\left(T_{1}^{5}+T_{2}^{5}\right) \\
+ & T_{\text {ext }} T_{2}\left(4 T_{1}^{8}+13 T_{1}^{5} T_{2}^{3}-16 T_{1}^{3} T_{2}^{5}-7 T_{2}^{8}\right) \\
& -T_{1} T_{2}^{5}\left(32 T_{1}^{3} T_{2}+8 T_{2}^{4}-24 T_{1}^{4}\right)=0 .
\end{aligned}
$$

Similarly to (15), the only unknown variable in this equation is $T_{2}$. Following a similar numerical procedure as in the case of maximum power conditions, we can calculate a convective cell structure. In Table 2 we present the numerical results for the maximum ecological function. We can see in Table 2 that with 16 successive Carnotian convective cells we can reach a final $T_{2} \approx 6000 \mathrm{~K}$.

Our results in Table 2 again show that the width of the cells decrease with increasing radius. The total width in this case is around $0.2859 R_{s}$ which is practically the value 0.286 $R_{s}$ given by other sun models [26].

A remarkable fact observed in Tables 1 and 2 (third column) is that between the cell number 10 and 16, the vertical linear sizes are between $2247 \mathrm{Km}$ and $247 \mathrm{Km}$, respectively. These are values near to those reported for the linear sizes of granules in [25], which are typically around 900-1000 Km, reaching their largest values up to $2000 \mathrm{Km}$ in diameter. On the other hand, in the highest convective cell of our model, the average power has a value of $5.7 \times$ $10^{9} \mathrm{erg} / \mathrm{cm}^{2} \mathrm{~s}$, which is of the order of the power reported in [25] for convection in the photosphere (which is $7 \times$ $10^{9} \mathrm{erg} / \mathrm{cm}^{2} \mathrm{~s}$ ). Our highest cell overlaps with photosphere. This result is also of the order of the power reported for a mixing length theory of convection in [25], which is 10-20 $\times$ $10^{9} \mathrm{erg} / \mathrm{cm}^{2} \mathrm{~s}$. Clearly, our oversimplified model coincides with those reported in [25] in that the energy transported by convection must increase rapidly as we go below the surface region of the convective zone. Finally, it is very interesting that all 16 cells in Tables 1 and 2 have practically the same thermal efficiency, $\eta \approx 0.307$.

\section{Concluding Remarks}

In the present work we have used a simplified finite-time thermodynamic method to describe the global thermal properties of the convective zone of the sun. This method was previously used by Gordon and Zarmi to describe convective 
TABle 2: Maximum ecological regime case: first column shows the normalized radial position of the hot layers corresponding to the sixteen virtual convective cells. The following columns give, respectively, second, the cell's widths; third, the hot isotherms; fourth, the cold isotherms; fifth, the average power output; sixth, thermal efficiency.

\begin{tabular}{lcccccc}
\hline No. & $r\left(T_{1}\right) / R_{s}$ & $\Delta r=r\left(T_{2}\right)-r\left(T_{1}\right)(\mathrm{Km})$ & $T_{1} \times 10^{6}(\mathrm{~K})$ & $T_{2} \times 10^{6}(\mathrm{~K})$ & $\bar{W}\left(\mathrm{erg} / \mathrm{cm}^{2} \cdot \mathrm{s}\right)$ & $\eta=\eta\left(T_{1}, T_{2}\right)$ \\
\hline 1 & 0.714 & 61370.8 & 2.187761 & 1.51504 & $6.49519 \times 10^{17}$ & 0.307494 \\
2 & 0.802176 & 42499.5 & 1.51504 & 1.04917 & $4.65788 \times 10^{17}$ & 0.307494 \\
3 & 0.863239 & 29431.1 & 1.04917 & 0.726558 & $1.07123 \times 10^{17}$ & 0.307494 \\
4 & 0.905525 & 20381.2 & 0.726558 & 0.503147 & $2.46362 \times 10^{16}$ & 0.307493 \\
5 & 0.934808 & 14114.1 & 0.503147 & 0.348433 & $5.66589 \times 10^{15}$ & 0.307492 \\
6 & 0.955087 & 9774.07 & 0.348433 & 0.241293 & $1.30306 \times 10^{15}$ & 0.307491 \\
7 & 0.96913 & 6768.59 & 0.241293 & 0.167098 & $2.99682 \times 10^{14}$ & 0.307489 \\
8 & 0.978855 & 4687.28 & 0.167098 & 0.115718 & $6.89224 \times 10^{13}$ \\
9 & 0.98559 & 3245.97 & 0.115718 & 0.0801366 & $1.58513 \times 10^{13}$ \\
10 & 0.990254 & 2247.85 & 0.0801366 & 0.0554965 & $3.64566 \times 10^{12}$ & 0.307482 \\
11 & 0.993483 & 1556.65 & 0.0554965 & 0.0384331 & $8.38504 \times 10^{11}$ \\
12 & 0.99572 & 1077.98 & 0.0384331 & 0.0266166 & $1.9288 \times 10^{11}$ & 0.307476 \\
13 & 0.997269 & 746.509 & 0.0266166 & 0.0184336 & $4.43885 \times 10^{10}$ & 0.307456 \\
14 & 0.998341 & 516.961 & 0.0184336 & 0.0127669 & $1.02346 \times 10^{10}$ & 0.307439 \\
15 & 0.999084 & 357.998 & 0.0127669 & 0.00884262 & $2.37873 \times 10^{9}$ & 0.307414 \\
16 & 0.999599 & 247.916 & 0.00884262 & 0.00612506 & $5.71627 \times 10^{8}$ & 0.307326 \\
\hline
\end{tabular}

motions of the air in the earth's atmosphere. These authors assert that this FTT-approach corresponds very approximately to the global scale motion of the wind in convective cells. However, it is necessary to remark that convective cells of this kind of FTT-models are only virtual cells performing by unit area and yearly averages. Thus, they only represent the global thermodynamic properties stemming from the first and second laws of thermodynamics; that is, kind of thermodynamically equivalent cells that only captures global average quantities and discards any other dynamical detail. Nevertheless, all these simplifications permit to obtain reasonable values for some thermal quantities associated to the convective zone of the sun. Our simplification is mainly based in taking several spherical virtual layers as black-body radiant surfaces, whose emitted radiation is rapidly absorbed by the opaque gases of the convective zone. This radiant energy is taken as the driver energy of convective cells.

\section{Acknowledgments}

This work was supported in part by CONACYT, COFAA, and EDI-IPN-México.

\section{References}

[1] M. Gordon and Y. Zarmi, "Wind energy as a solar-driven heat engine: a thermodynamic approach," American Journal of Physics, vol. 57, pp. 995-998, 1989.

[2] A. De Vos and G. Flater, "The maximum efficiency of the conversion of solar energy into wind energy," American Journal of Physics, vol. 59, pp. 751-754, 1991.

[3] A. De Vos, Endoreversible Thermodynamics of Solar Energy Conversion, Oxford University Press, Oxford, UK, 1992.
[4] A. De Vos and P. van del Wel, "Endoreversible models of the conversion of solar energy into wind energy," Journal of NonEquilibrium Thermodynamics, vol. 17, pp. 77-89, 1992.

[5] A. De Vos and P. van der Wel, "The efficiency of the conversion of solar energy into wind energy by means of Hadley cells," Theoretical and Applied Climatology, vol. 46, no. 4, pp. 193202, 1993.

[6] M. A. Barranco-Jiménez and F. Angulo-Brown, "A nonendoreversible model for wind energy as a solar-driven heat engine," Journal of Applied Physics, vol. 80, no. 9, pp. 4872-4876, 1996.

[7] M. A. Barranco-Jiménez and F. Angulo-Brown, "A simple model on the influence of the greenhouse effect on the efficiency of solar-to-wind energy conversion," Il Nuovo cimento della Società Italiana di Fisica, vol. 26, no. 5, p. 235, 2003.

[8] M. A. Barranco-Jiménez, J. C. Chimal-Eguía, and F. AnguloBrown, "The Gordon and Zarmi model for convective atmospheric cells under the ecological criterion applied to the planets of the solar system," Revista Mexicana de Fisica, vol. 52, no. 3, pp. 205-212, 2006.

[9] A. Raval and V. Ramanathan, "Observational determination of the greenhouse effect," Nature, vol. 342, no. 6251, pp. 758-761, 1989.

[10] M. H. Rubin, "Optimal configuration of a class of irreversible heat engines-I," Physical Review A, vol. 19, no. 3, pp. 12721276, 1979.

[11] F. Angulo-Brown, "An ecological optimization criterion for finite-time heat engines," Journal of Applied Physics, vol. 69, no. 11, pp. 7465-7469, 1991.

[12] F. Curzon and B. Ahlborn, "Efficiency of a Carnot engine at maximum power output," American Journal of Physics, vol. 43, no. 1, p. 22, 1975.

[13] L. A. Arias-Hernández and F. Angulo-Brown, "A general property of endoreversible thermal engines," Journal of Applied Physics, vol. 81, no. 7, pp. 2973-2979, 1997. 
[14] B. W. Carrol and D. A. Ostlie, An Introduction to Modern Astrophysics, Pearson-Adisson Wesley, San Francisco, Calif, USA, 2007.

[15] D. Gutkowicz-Krusin, I. Procaccia, and J. Ross, "On the efficiency of rate processes. Power and efficiency of heat engines," The Journal of Chemical Physics, vol. 69, no. 9, pp. 3898-3906, 1978.

[16] M. R. Gustavson, "Limits to wind power utilization," Science, vol. 204, no. 4388, pp. 13-17, 1979.

[17] A. Curry and P. J. Wester, Thermodynamics of Atmospheres \& Oceans, International Geiphysics Series, Academic Press, 1999.

[18] E. Lorentz, Dynamic of Climate, vol. 86, Pergamon Press, New York, NY, USA, 1960.

[19] L. Schulman, "A theoretical study of the efficiency of the general circulation," Journal of the Atmospheric Sciences, vol. 34, pp. 559-580, 1977.

[20] S. R. Caplan and A. Essig, Bioenergectics and Linear Nonequilibrium Thermodynamics, Harvard University Press, Cambridge, Mass, USA, 1983.

[21] F. Angulo-Brown, M. Santillán, and E. Calleja-Quevedo, "Thermodynamic optimality in some biochemical reactions," Il Nuovo Cimento D, vol. 17, no. 1, pp. 87-90, 1995.

[22] F. Angulo-Brown, L. A. Arias-Hernández, and M. Santillán, "On some connections between first order irreversible thermodynamics and finite-time thermodynamics," Revista Mexicana de Fisica, vol. 48, pp. 182-192, 2002.

[23] M. Santillán, L. A. Arias-Hernández, and F. Angulo-Brown, "Some optimization criteria for biological systems in linear irreversible thermodynamics," Il Nuovo cimento della Società Italiana di Fisica D, vol. 19, no. 1, pp. 99-109, 1997.

[24] Encyclopaedia Britannica, vol. 2, William Benton Publisher, 15th edition, 1991.

[25] D. J. Mullan, Physics of the Sun: A First Course, CRC Press, Boca Raton, Fla, USA, 2010.

[26] P. A. Sturrock, T. E. Holzer, D. M. Mihalas, and R. K. Ulrich, "Physics of the Sun, Vol. I: The solar interior," B. M. McCormac, Ed., Loockheed Palo Alto Research Laboratory, Calif, USA, 1986. 

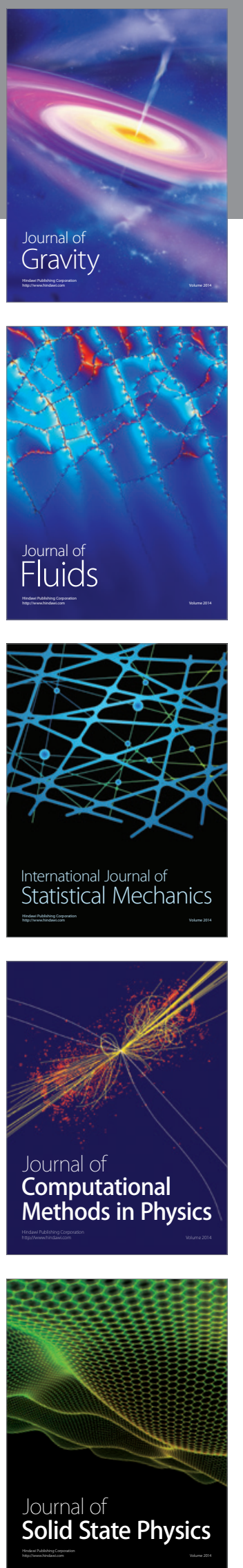

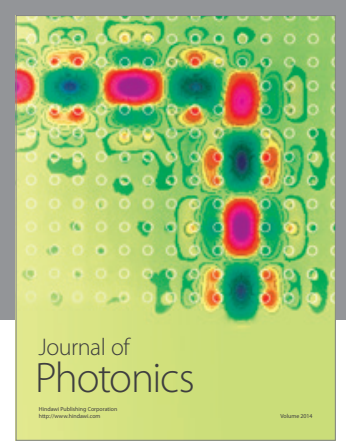

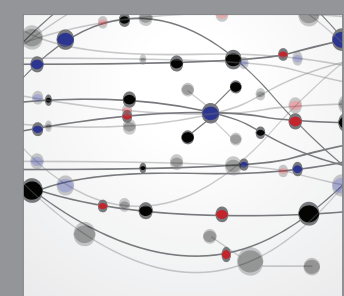

The Scientific World Journal
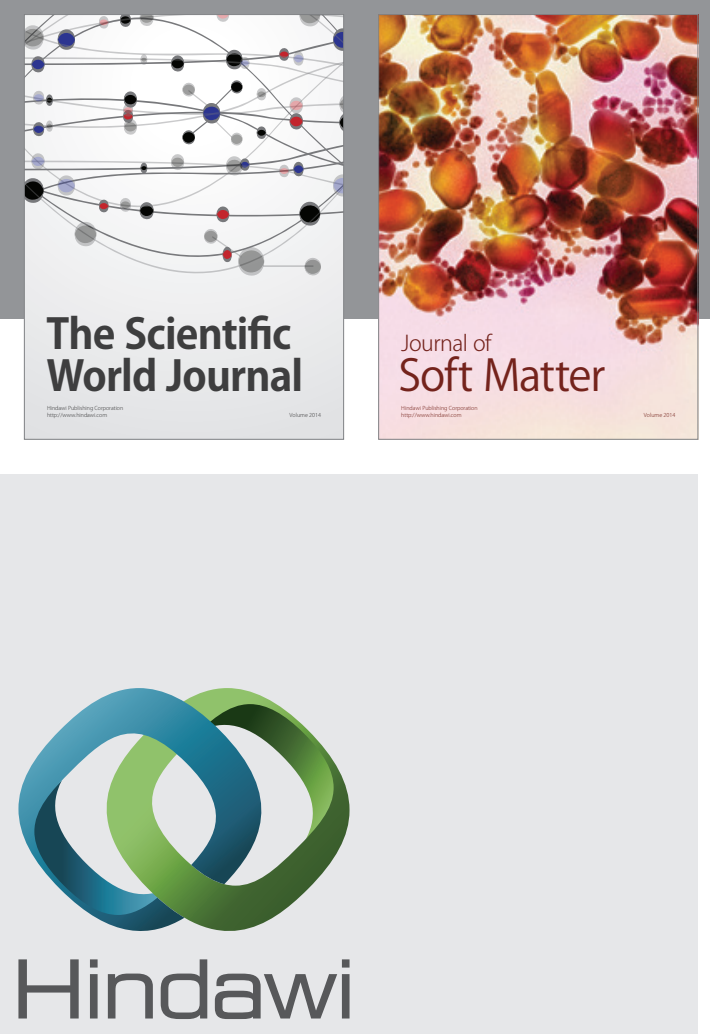

Submit your manuscripts at

http://www.hindawi.com
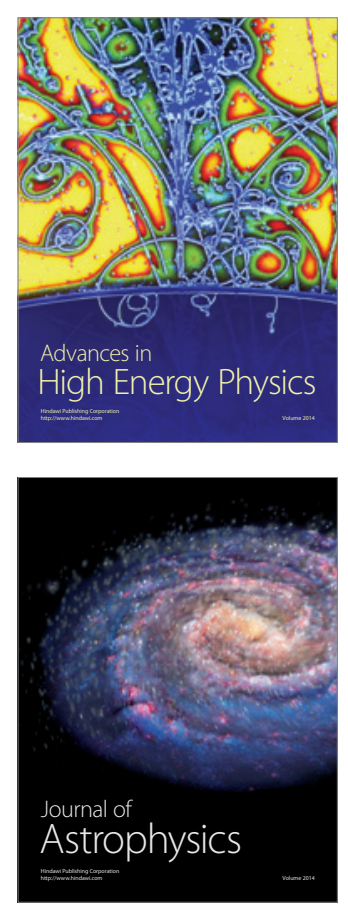
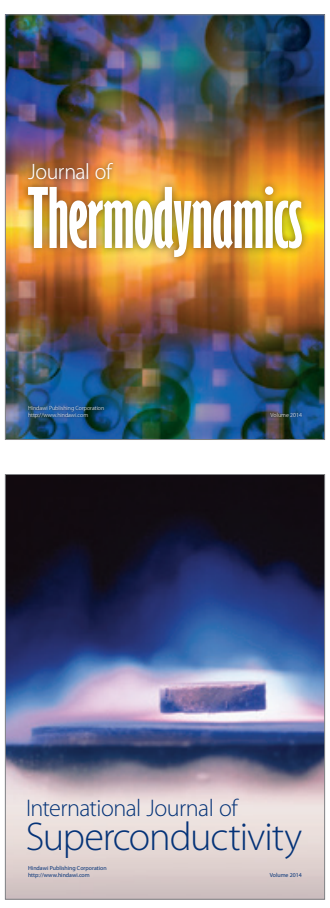
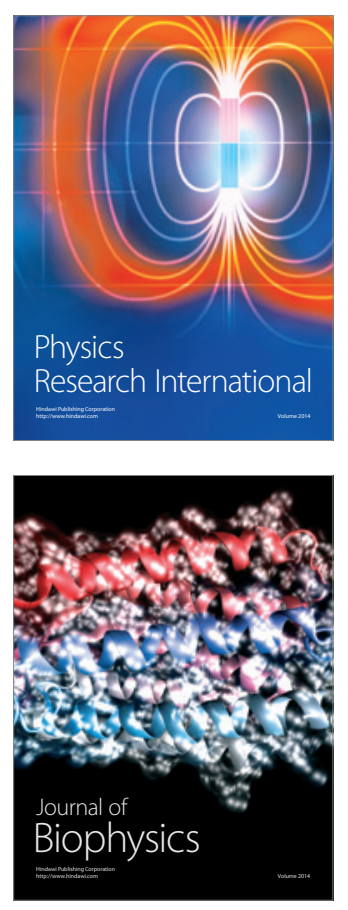
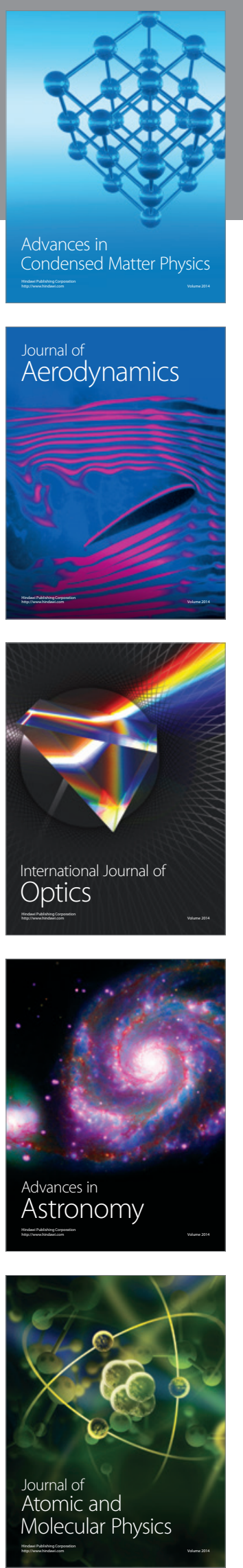